\title{
Some Orthogonal Polynomials on the Finite Interval and Gaussian Quadrature Rules for Fractional Riemann-Liouville Integrals
}

\author{
Gradimir Milovanovic ${ }^{1}$ \\ ${ }^{1}$ Serbian Academy of Sciences and Arts
}

June 19, 2020

\begin{abstract}
Inspired by papers by M.A. Bokhari, A. Qadir, and H. Al-Attas [On Gauss-type quadrature rules, Numer. Funct. Anal. Optim. 31 (2010), 1120-1134] and by M.R. Rapaic, T.B. Sekara, and V. Govedarica [A novel class of fractionally orthogonal quasipolynomials and new fractional quadrature formulas, Appl. Math. Comput. 245 (2014), 206-219], in this paper we investigate a few types of orthogonal polynomials on finite intervals and derive the corresponding quadrature formulas of Gaussian type for efficient numerical computation of the left and right fractional Riemann-Liouville integrals. Several numerical examples are included to demonstrate the numerical efficiency of the proposed procedure.
\end{abstract}

\section{Hosted file}

gvm-2020.pdf available at https://authorea.com/users/335199/articles/461056-some-orthogonalpolynomials-on-the-finite-interval-and-gaussian-quadrature-rules-for-fractional-riemannliouville-integrals 
figures/wBa/wBa-eps-converted-to.pdf 
figures/Fxcs/Fxcs-eps-converted-to.pdf 
figures/fxcf/fxcf-eps-converted-to.pdf 
figures/wL05do10/wL05do10-eps-converted-to.pdf 
figures/bkoef 1002/bkoef 1002-eps-converted-to.pdf 
figures/bkoef 1006/bkoef 1006-eps-converted-to.pdf 
figures/EX1new/EX1new-eps-converted-to.pdf 
figures/grn5GL02505/grn5GL02505-eps-converted-to.pdf 
figures/compGL02505/compGL02505-eps-converted-to.pdf 
figures/EX1sla14b1/EX1sla14b1-eps-converted-to.pdf 
figures/slika13/slika13-eps-converted-to.pdf 
figures/EX1sla12b14/EX1sla12b14-eps-converted-to.pdf 
figures/ex $2 a n 5 / e x 2 a n 5-e p s-c o n v e r t e d-t o \cdot p d f$ 
figures/Ex2aA025/Ex2aA025-eps-converted-to.pdf 
figures/por025n5SKRtext/por025n5SKRtext-eps-converted-to.pdf 
figures/poraj025/poraj025-eps-converted-to.pdf 
figures/FrInt3/FrInt3-eps-converted-to.pdf 
figures/EX3GuRada025b05/EX3GuRada025b05-eps-converted-to.pdf 